\title{
A Critical Role for Human Ventromedial Frontal Lobe in Value Comparison of Complex Objects Based on Attribute Configuration
}

\author{
(․)Gabriel Pelletier and 는 Kesley Kellows \\ Montreal Neurological Institute, Department of Neurology \& Neurosurgery, McGill University, Montreal, Quebec H3A 2B4, Canada
}

In making decisions, we often choose from among options with multiple value-relevant attributes. Neuroeconomic models propose that the value associated with each attribute is integrated in a global value for each option. However, some evidence from patients with ventromedial frontal lobe (VMF) damage argues against a very general role for this region in value integration, suggesting instead that it contributes critically to a specific value inference or comparison process. Here, we tested value-based decision-making involving artificial multiattribute objects in humans with focal damage to the VMF $(N=12)$ compared with a healthy group matched for age and education $(N=24)$ and with a group with frontal lobe damage sparing the VMF $(N=12)$. In a "configural" condition, overall object value was predicted by the conjunction of two attributes, while in an "elemental" condition, object value could be assessed by combining the independent values of individual attributes. Patients with VMF damage were impaired in making choices when value was uniquely predicted by the configuration of attributes, but intact when choosing based on elemental attribute values. This is evidence that the VMF is critical for inferring the value of whole objects in a multiattribute choice. These findings have implications for models of value-based choice and add to emerging views of how this region may interact with medial temporal lobe systems involved in configural object processing and relational memory.

Key words: decision-making; learning; lesion; neuropsychology; reward

Significance Statement

Neuroeconomic models propose that the ventromedial frontal lobe (VMF) supports multiattribute decisions by integrating the values of attributes. However, researchers have been uncertain about the underlying mechanisms for this process. Patients with VMF damage made multiattribute choices under two conditions: in one, attribute values could be summed to guide choice; in the other, value was predicted by the conjunction of attributes. VMF damage impaired only the latter. This argues that the VMF is critical for inferring value from configural information to guide multiattribute object choice. This region may be key for judging the emergent "value of the forest," rather than for integrating the individual "value of each tree."

\section{Introduction}

In making decisions, we often choose among options with multiple attributes. For instance, snacks can be characterized on taste, price, and healthiness. Individual attributes might directly predict subjective value: if one craves sweets, chocolate will be valued over peanuts. However, value can also emerge from the interac-

\footnotetext{
Received Nov. 20, 2018; revised Feb. 5, 2019; accepted March 4, 2019.

Author contributions: G.P. and L.K.F. designed research; G.P. performed research; G.P. and L.K.F. analyzed data;

G.P. wrote the first draft of the paper; G.P. and L.K.F. edited the paper; G.P. and L.K.F. wrote the paper.

The authors declare no competing financial interests.

This work was supported by the Canadian Institutes of Health Research (Grant MOP 249071) and the Natural Sciences and Engineering Research Council of Canada (RGPIN 2016-06066). We thank Christine Déry for her help with participant recruitment. We also thank the patients and healthy volunteers for their participation.

Correspondence should be addressed to Gabriel Pelletier at gabriel.pelletier@mail.mcgill.ca.

https://doi.org/10.1523/JNEUROSCI.2969-18.2019

Copyright $\odot 2019$ the authors
}

tion of attributes. For example, for those who enjoy sucré-salé flavors, the combination of peanuts and chocolate in the same snack would yield a value greater than the sum of the value of each attribute.

Neuroeconomic models propose that subjective value is encoded in a common currency within the ventral prefrontal cortex (Bartra et al., 2013). The overall value of objects composed of multiple value-predictive attributes (e.g., colors and shapes associated with monetary rewards) can be decoded from spatially distributed patterns of BOLD activity in the human ventromedial prefrontal cortex (vmPFC; Kahnt et al., 2011). Researchers have likewise found within the vmPFC or adjacent medial orbitofrontal cortex (OFC) signals reflecting value derived from the nutrient components of food items (Suzuki et al., 2017), monetary costs and benefits (Basten et al., 2010), and esthetic and semantic 


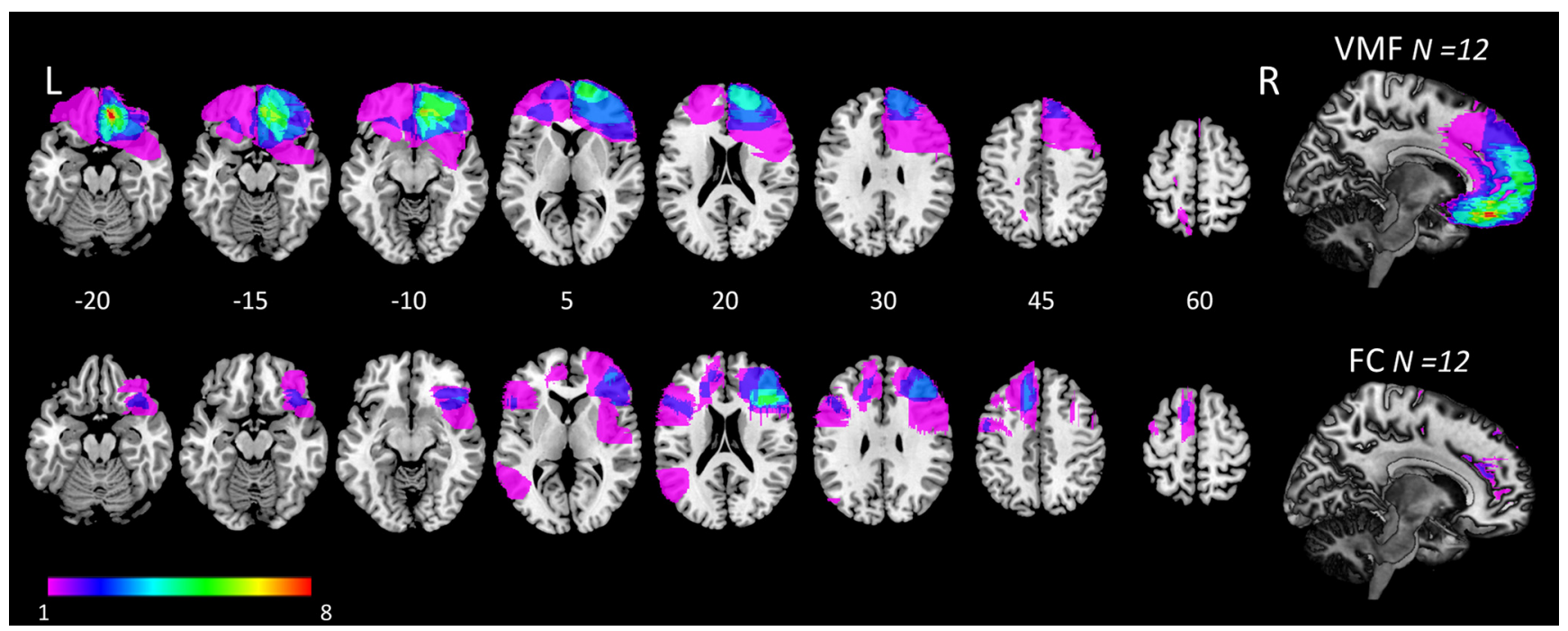

Figure 1. Lesion overlap in the VMF (top row) and FC (bottom row) groups. Colors indicate extent of lesion overlap, as shown in the legend. Numbers indicate axial slices by z-coordinate in Montreal Neurological Institute space. L, Left; R, Right.

aspects of T-shirt graphics (Lim et al., 2013). In nonhuman primates, activity in the OFC correlates with the subjective value of juice options varying in taste and quantity (Padoa-Schioppa and Assad, 2006). These findings have been taken as evidence that multiple-attribute values are integrated into an overall option value representation within the vmPFC/OFC, subsequently influencing choice (Levy and Glimcher, 2012).

However, direct evidence that this region is necessary for value integration is lacking. Damage to the vmPFC and adjacent OFC [together termed "ventromedial frontal lobe" (VMF)] has been shown to change how multiattribute information (presented as text) is acquired during decision-making (Fellows, 2006), and to affect which attributes (inferred from faces) influence choice (Xia et al., 2015). Compared with healthy and other frontal-damaged individuals, people with VMF damage differed in how they weighted specific attributes when evaluating visual art (Vaidya et al., 2018). These observations could be consistent with a deficit in attribute-value integration, as predicted by value-integration models. Puzzlingly, however, these VMF-damaged patients seemed to systematically neglect specific attributes, rather than showing a generic reduction in the attributes that were considered. Other recent work found that VMF damage impaired value-based decisions about spouses, but not houses, and that the number of attributes per option did not affect choice consistency in either condition (Bowren et al., 2018). Together, this argues against a general role for the VMF in combining attributes to assess value, calling instead for a more specific account. One possibility is that this region is required for inferring value from the configural relationship among multiple lower-level attributes. In our opening example, the VMF would thus be required to predict the unique value of peanuts and chocolate together, and perhaps not for summing the individual values of each of those attributes alone.

This hypothesis was inspired by object-processing research showing that encoding of individual attributes and the conjunctions of attributes is supported by partly dissociable neural substrates. Configural processes rely on the medial temporal lobe (MTL): damage to the hippocampus impairs working memory for object-location configurations (Olson et al., 2006) and object-outcome associative learning predicted by attribute configurations (Rudy and Sutherland, 1995). The perirhinal cortex represents complex objects distinct from the combined representations of their parts (Erez et al., 2016), and damage to this region impairs object discrimination based on configurations (Bussey et al., 2005). In contrast, attribute-outcome associations and object discrimination based on individual object parts do not rely on an intact MTL. There are both strong anatomical connections (Von Der Heide et al., 2013) and evidence of functional connectivity (Andrews-Hanna et al., 2014; Eichenbaum, 2017) between the MTL and VMF. These regions may interact during decision-making (McCormick et al., 2018; Gluth et al., 2015). Thus, how attributes of complex objects are represented in the MTL may be relevant to understanding the role of the VMF in assigning value to such objects.

Here, we tested the hypothesis that the VMF plays a specific role in inferring value from multiattribute configurations. We asked whether VMF damage impairs decisions for choosing objects when values were predicted by attribute configurations, by the integration of individual attribute values, or both.

\section{Materials and Methods}

Participants. Twenty-four patients with focal frontal lobe damage were recruited through the cognitive neuroscience research database at McGill University. All patients with a fixed lesion primarily affecting the frontal lobes were eligible. Lesions were characterized with magnetic resonance or computed tomography imaging, and registered manually, using MRIcro software, to the Montreal Neurological Institute standard brain by a neurologist blind to task performance (Rorden and Brett, 2000; www. mccauslandcenter.sc.edu/crnl/mricro). Patients were assigned a priori to a group with damage involving the VMF, the region of interest in this study (VMF, $N=12$ ) or a frontal comparison group with damage sparing the VMF (FC, $N=12$ ). Figure 1 depicts lesion extent and overlap for the VMF and FC groups. In the VMF group, damage was unilateral in 11 of 12 cases (eight right, three left) and bilateral in one case. By design, VMF lesions affected the medial OFC in all cases to some degree. VMF damage extended to adjacent ventral regions in most cases, most commonly the vmPFC (in eight) and lateral OFC (in four). In six of $12 \mathrm{VMF}$ patients, damage extended into the medial frontal lobe superior to the genu of the corpus callosum. Such dorsomedial damage was also present in four of 12 FC patients. Twenty-four healthy comparison (HC) participants matched for age and education were also recruited from a companion database that drew participants from the Montreal area via 


\begin{tabular}{|c|c|c|c|c|c|c|c|c|c|c|}
\hline \multirow[b]{2}{*}{ Group } & \multirow[b]{2}{*}{$N$} & \multirow[b]{2}{*}{ Age (years) } & \multirow[b]{2}{*}{ Sex (male/female) } & \multirow[b]{2}{*}{$\begin{array}{l}\text { Handedness } \\
\text { (right/left/ambidextrous) }\end{array}$} & \multirow[b]{2}{*}{$\begin{array}{l}\text { Education } \\
\text { (years) }\end{array}$} & \multicolumn{2}{|l|}{ HADS } & \multirow[b]{2}{*}{$\begin{array}{l}\text { estimated } \\
\mathrm{IQ}^{a}\end{array}$} & \multirow[b]{2}{*}{$\begin{array}{l}\text { Lesion laterality } \\
\text { (right/left/bilateral) }\end{array}$} & \multirow[b]{2}{*}{$\begin{array}{l}\text { Lesion } \\
\text { volume (cc }\end{array}$} \\
\hline & & & & & & $\begin{array}{l}\text { Anxiety } \\
\text { scale }\end{array}$ & $\begin{array}{l}\text { Depression } \\
\text { scale }\end{array}$ & & & \\
\hline $\mathrm{HC}$ & 24 & $61(11.6)$ & $7 / 17$ & $23 / 1 / 0$ & $16(3.0)$ & $3.8(3.0)$ & $2.2(2.1)$ & $126(4)$ & - & - \\
\hline $\mathrm{FC}$ & 12 & $60(10.7)$ & $5 / 7$ & $10 / 2 / 0$ & $15.1(2.9)$ & $5.8(4.0)$ & $5.1(3.6)^{*}$ & $120(10)$ & $6 / 6 / 0$ & $24(5-37)$ \\
\hline
\end{tabular}

All values mean (SD), except sex (count) and lesion volume [median (range)]. ${ }^{*} p<0.05$, Mann-Whitney $U$ test compared to healthy controls.

${ }^{a}$ Not all subjects completed the estimated IQ test $(\mathrm{HC}, N=11 ; \mathrm{VMF}, N=7 ; \mathrm{FC}, N=6)$.

community advertisement. Healthy controls scored $>26$ on the Montreal Cognitive Assessment and denied any current psychiatric or neurologic diagnosis or the use of psychoactive medications.

Damage to the VMF was caused by aneurysm in two cases, hemorrhagic stroke in one case, and tumor resection in nine cases. Damage in the FC group was caused by ischemic stroke in six cases, hemorrhagic stroke in two cases, and tumor resection in four cases. Nine patients (seven VMF, two FC) were taking one or more psychoactive drugs, most commonly an antidepressant or anticonvulsant. All patients had fixed, circumscribed lesions of $\geq 6$ months duration (mean, 8.3 years; SD, 4.9 years; Table 1).

Neuropsychological characterization. Participants with frontal lobe damage completed tests of working memory (backwards digit span; Lezak et al., 2012), verbal fluency (Animal, Fluency-F; Benton et al., 1989), language comprehension (similar to the Token test; De Renzi and Vignolo, 1962), and incidental memory for faces (Bower and Karlin, 1974).

Apparatus. All HC participants and 20 patients were tested in the laboratory on a desktop computer equipped with a 19-inch monitor. Four participants with frontal damage (three VMF, one FC) were tested at home using a 15-inch laptop computer (Fujitsu). Subjects responded using a standard mouse or keyboard, depending on the task. Experiments were programmed in Matlab (version 2014b, Mathworks), using the Psychtoolbox extension (PTB-3; Brainard, 1997).

Experimental tasks. Participants made value-based decisions between multiattribute objects in two conditions, elemental and configural. In the configural condition, participants learned the value of unique configurations of two attributes of an object, whereas in the elemental condition, participants learned the values of individual attributes, which then had to be combined for optimal multiattribute decisions. Participants also completed two control tasks to assess object discrimination and memory for single attribute-value associations over a delay.

We used novel multiattribute stimuli developed to study object processing. These pseudo-objects, called "fribbles," are composed of a main body and four appendages, each taking one of three possible forms, referred to here as "attributes." They were designed to mimic perceptual characteristics of real-world objects (Williams and Simons, 2000; Barry et al., 2014). To familiarize participants with these novel stimuli and establish that VMF damage did not affect the ability to discriminate fribbles, the session began with a discrimination task adapted from a previous study on complex object perception in patients with MTL damage (Barense et al., 2007). The task was divided into two parts. In the first 12 trials, three fribbles were displayed side by side: two were identical; one was different. All fribbles had the same main body and three of four attributes in common, such that the "odd fribble out" was distinguished by a single attribute. The participants were asked to select the fribble that was different. Once the response was registered (by a mouse click), feedback was given by surrounding the selected fribble with a green (correct) or red (error) border for $1.5 \mathrm{~s}$ before proceeding to the next trial. The second 12 trials followed the same procedure but five fribbles were presented: two pairs of identical fribbles and one fribble that could not be paired with any other. Again, participants were instructed to click on the odd fribble out. Importantly, the odd fribble out shared all its attributes with at least two other fribbles in the set, such that it could only be identified based on the specific configuration (i.e., conjunction) of two attributes.

The main task had a learning phase followed by a decision phase for each of two conditions: elemental and configural. Participants learned a total of six fribble-value associations in two sets of three by observing the outcomes of mock auctions as fribbles were "sold," one at a time. Participants were instructed to carefully study the different fribbles and the price for which each was sold. A learning trial started with the presentation of a fribble. After a $2 \mathrm{~s}$ delay, the amount for which the item had been sold was presented (Fig. 2). The fribble and its selling price were displayed until the participant pressed a key to go to the next trial. One learning block included three different fribbles, presented nine times each in random order for a total of 27 trials. The selling value associated with a fribble on a given trial was randomly drawn from a normal distribution with an SD of $\$ 5$; median values are shown in Figure 2. The fribble associated with each value was randomized, counterbalanced across participants.

After each learning block, learning was assessed with a binary choice probe. On each trial, two fribbles were presented on the screen to the left and right of a central fixation cross (Fig. 2). Participants were asked to select with the corresponding arrow key which of the two fribbles they thought was worth the most. The response was coded as an error if the less valuable option was chosen, but no feedback was given to the participant. A learning probe block had a maximum of 36 trials ( 12 repetitions of the three pairs), but was stopped sooner if the learning criterion was violated. Learning blocks and probes were repeated until a criterion of $92 \%$ (11 of 12) correct for each of the three pairs was reached. When the criterion was reached with the first set of fribbles, participants were trained on a second set of three following the same procedure.

After learning, participants completed a decision phase that drew upon all six learned associations. On each trial, two fribbles were presented on the screen and participants were instructed to choose which fribble they wanted to have in their inventory (Fig. 2C). Participants were told that each fribble they chose would be placed in their inventory, and that this inventory would be sold at the end of the experiment with the proceeds converted into real money (maximum \$7) and added to their base compensation for participation.

In the configural condition, participants learned the value of six fribbles having the same body and two (irrelevant) attributes in common (Fig. 2). Only the configuration of the two varying attributes was valuepredictive. During the decision phase, all 15 possible pairs were presented six times in random order for a total of 90 trials. In the elemental condition, values were associated with individual attributes. During the learning phase, the fribble body and irrelevant attributes were masked with a $50 \%$ transparent white mask, making the individual value-predictive attribute more salient (Fig. 2). Participants were explicitly told that the auction value was associated only with the unmasked attribute during the learning blocks, and only that attribute was relevant for the options' value during the learning probes. During the decision phase, stimuli were presented without masks, so all attributes were equally salient, and participants were instructed to take into account everything they had learned about the different parts. The stimulus set included nine different fribbles $(3 \times 3$ attributes $)$. Thirty-six possible pairs were presented five times each, in random order, for a total of 180 trials. Half the trials involved choices between fribbles distinguished by one attribute only (the other attribute being common to the two options), referred to as "singleattribute trials." Half the trials involved choices between fribbles for which both value-predicting attributes were varied, referred to as "twoattribute trials." In principle, the more valuable fribble in these trials could be selected by combining (e.g., adding) individual attribute values, as each attribute was associated with a specific value, regardless of which 
A
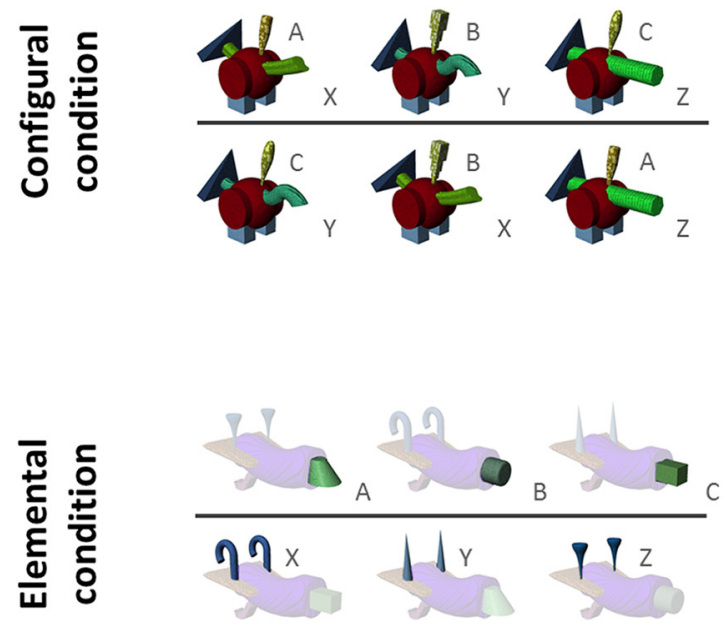

\begin{tabular}{c|c} 
Attribute & Value (\$) \\
\hline A & 13 \\
B & 25 \\
C & 37 \\
X & 13 \\
Y & 25 \\
Z & 37
\end{tabular}

B

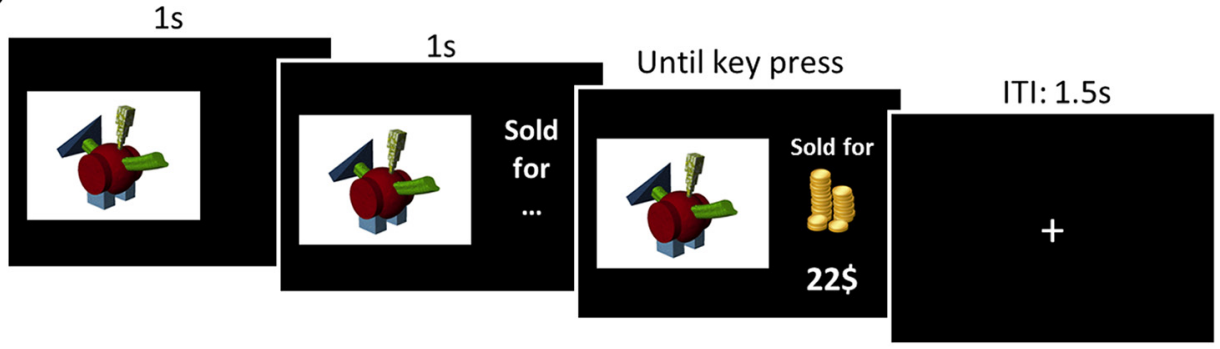

C

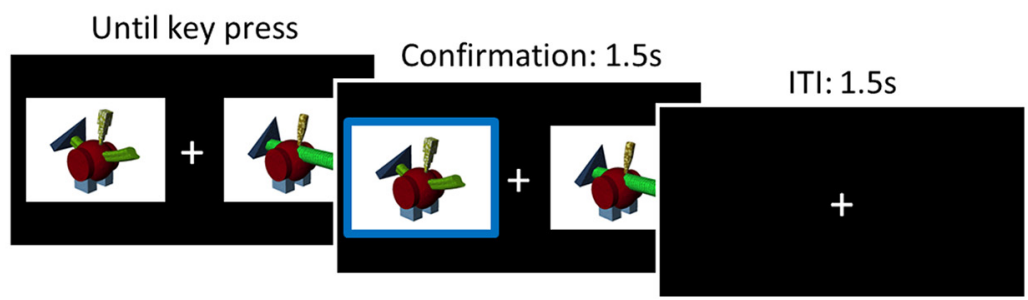

Figure 2. Stimulus sets and experimental paradigm. $A$, Example of stimulus sets and value associations. Stimulus sets were counterbalanced across participants and stimulus-value associations were randomly selected from six predefined lists. $\boldsymbol{B}$, Structure of a learning trial. A fribble was displayed for $2 \mathrm{~s}$. Then its selling price was presented until a key was pressed to move to the next trial, following a $1.5 \mathrm{~s}$ intertrial interval. C, Binary choice trial (for learning probes and final decision phases). These were self-paced: two fribbles were presented on either side of a fixation cross and participants pressed the left or right arrow key to choose which item they wanted in their inventory. Choice was confirmed with a bold border surrounding the chosen object for $1.5 \mathrm{~s}$, followed by a 1.5 s intertrial interval.

other attributes were present. To eliminate the possibility of an attributevalue "task set" interfering with learning the values of attribute configurations, all participants completed the configural condition first. Stimulus sets were counterbalanced across conditions and participants.

In both the elemental and configural conditions, stimulus-value associations were learned in two different sets before the decision phase. Thus, half the associations called upon in the decision phase were learned more recently. A control task was therefore included to determine whether there were groupwise differences in retaining stimulus-value associations across this delay. This task used a new set of fribbles and was completed after the decision tasks. Three attribute-value associations were trained to criterion, as in the learning phase of the elemental condition. This was followed by an unrelated task (Posner cueing task) lasting $\sim 10 \mathrm{~min}$, after which memory for the learned associations was probed with a series of binary decisions, identical to the learning probe blocks described earlier.

Statistical analysis. Task performance was assessed through accuracy and reaction times. Correct responses were defined as choices of the higher-value fribble in each pair. In the configural condition, each option's value was the mean value associated with the specific configuration of attributes during training. For the elemental condition, we defined each option's compound value by summing the mean value of each attribute (learned during training), although any method of combining the two learned values with equal weights would lead to the same stimulus-value ranking. A choice of the option with the lower objectively determined value was coded as an error. Accuracy (percentage correct) data were arcsine transformed for statistical analysis, although using the raw data yielded the same pattern of results. 
Table 2. Neuropsychological screening test performance for patient groups

\begin{tabular}{|c|c|c|c|c|c|}
\hline \multirow[b]{2}{*}{ Group } & \multicolumn{2}{|l|}{ Fluency } & \multirow[b]{2}{*}{$\begin{array}{l}\text { Backwards } \\
\text { digit span }\end{array}$} & \multirow{2}{*}{$\begin{array}{l}\text { Incidental } \\
\text { memory } \\
\text { (accuracy) }\end{array}$} & \multirow{2}{*}{$\begin{array}{l}\text { Sentence } \\
\text { comprehension } \\
\text { (accuracy) }\end{array}$} \\
\hline & $\begin{array}{l}\text { Animals, } \\
60 \mathrm{~s}\end{array}$ & $\begin{array}{l}\text { Fluency-F, } \\
60 \mathrm{~s}\end{array}$ & & & \\
\hline VMF damage & $10.3(5.1)$ & $18.2(2.9)$ & $2.6(0.8)$ & $0.88(0.1)$ & $0.99(0.02)$ \\
\hline $\mathrm{FC}$ & $10.5(5.4)$ & $17.7(6.7)$ & $2.9(1.3)$ & $0.79(0.1)$ & $0.96(0.07)$ \\
\hline
\end{tabular}

Mean (SD). One VMF and one FC participant did not complete the screening tests.

Unless otherwise specified, statistical analyses were run using IBM SPSS Statistics for Windows (version 22). Performance was compared across groups using ANOVA followed by Bonferroni-corrected pairwise comparisons where significant main effects were found. Generalized estimating equations (GEEs) were used to analyze the trial-by-trial influence of value on choice behavior using SAS (version 9.4, SAS Institute). This analysis is similar to binary logistic regression, but is better suited to modeling repeated measures where outcomes might be correlated within participants, such as in this case. The left-minus-right option value difference was used as a predictor to model the choice of the left option as a binary outcome. Demographic variables and neuropsychological test scores were compared between patient and HC groups using $t$ tests or, when assumptions for parametric analysis were not met, Mann-Whitney $U$ tests, without correction for multiple comparisons.

\section{Results}

\section{Participant characteristics}

Demographic and clinical information is reported in Table 1. There was no significant difference in age or years of education between groups. There was no significant group difference in premorbid IQ, estimated using the American National Reading Test (Grober and Sliwinski, 1991) at the time of enrolment in the registry (postlesion), although this measure was only available in a subset of participants. Patient groups did not differ in lesion volume. The Hospital Anxiety and Depression Scale (HADS) was used to characterize levels of anxiety and depression (Zigmond and Snaith, 1983). Scores on the depression scale (HADS-D) were higher in the FC group relative to the HC group $(p=0.03)$, but there was no significant difference between the VMF and HC groups $(p=0.29)$, nor between frontal groups $(p=0.26)$. There were no group differences in anxiety scores (HADS-A). No participant had an active clinically diagnosed mood disorder, by self-report or chart review. Neuropsychological test results are shown in Table 2. There were no significant differences between patient groups in tests of incidental memory for faces, verbal fluency, or language comprehension. Patient groups differed in lesion etiology $\left(\chi_{(3)}^{2}=13.04, p=0.004\right)$, but we did not find effects of etiology on any variable of interest. There was no significant difference between the patients tested at home and those tested in the laboratory on any variable of interest.

\section{Control tasks}

Performance of the control tasks assessing the ability to discriminate fribbles and the ability to retain attribute-value associations across a 10 min delay is presented in Table 3. All subjects could discriminate fribbles distinguished by a single attribute or by the conjunction of two attributes. There was no main effect of group on accuracy in the three-fribble (one-way ANOVA, $F_{(2,45)}=0.79$, $\left.p=0.46, \eta_{\mathrm{p}}^{2}=0.03\right)$ or five-fribble trials $\left(F_{(2,45)}=1.47, p=0.24\right.$, $\left.\eta_{\mathrm{p}}^{2}=0.06\right)$. There was a main effect of group on reaction times in the three-fribble trials $\left(F_{(2,45)}=4.82, p=0.01, \eta_{\mathrm{p}}^{2}=0.18\right)$. Post hoc comparisons revealed that the FC group was slower than the VMF group $(p=0.01)$, but neither patient group was different from the HC group (HC-VMF, $p=0.26$; HC-FC, $p=0.22$ ).
Table 3. Control task performance for HC and patient groups [mean (SD)]

\begin{tabular}{|c|c|c|c|c|c|c|}
\hline \multirow[b]{3}{*}{ Group } & \multicolumn{4}{|c|}{ Discrimination } & \multirow{2}{*}{\multicolumn{2}{|c|}{$\begin{array}{l}\text { Decision probe } \\
\text { after delay }\end{array}$}} \\
\hline & \multicolumn{2}{|c|}{ Three fribbles } & \multicolumn{2}{|l|}{ Five fribbles } & & \\
\hline & $\begin{array}{l}\text { Accuracy } \\
\text { (\% correct) }\end{array}$ & $\begin{array}{l}\text { Reaction } \\
\text { time (ms) }\end{array}$ & $\begin{array}{l}\text { Accuracy } \\
\text { (\% correct) }\end{array}$ & $\begin{array}{l}\text { Reaction } \\
\text { time (ms) }\end{array}$ & $\begin{array}{l}\text { Accuracy } \\
\text { (\% correct) }\end{array}$ & $\begin{array}{l}\text { Reaction } \\
\text { time (ms) }\end{array}$ \\
\hline $\mathrm{HC}$ & $99.0(2.8)$ & 4940 (1317) & $96.2(8.1)$ & 13,248 (6976) & $97.6(5.6)$ & 1387 (412) \\
\hline VMF damage & $99.3(2.4)$ & 4165 (667) & $91.0(13.5)$ & $11,043(3378)$ & $99.3(2.4)$ & $1288(220)$ \\
\hline $\mathrm{FC}$ & $100(0)$ & 5755 (1539) & $95.8(5.6)$ & $13,913(8766)$ & $97.9(5.2)$ & $1206(299)$ \\
\hline
\end{tabular}

Table 4. Attribute-value task-learning phase performance [mean (SD)]

\begin{tabular}{|c|c|c|c|c|}
\hline \multirow[b]{3}{*}{ Group } & \multicolumn{4}{|l|}{ Condition } \\
\hline & \multicolumn{2}{|l|}{ Configural } & \multicolumn{2}{|l|}{ Elemental } \\
\hline & $\begin{array}{l}\text { Number of } \\
\text { learning blocks }\end{array}$ & $\begin{array}{l}\text { Reaction } \\
\text { time (ms) }\end{array}$ & $\begin{array}{l}\text { Number of } \\
\text { learning blocks }\end{array}$ & $\begin{array}{l}\text { Reaction } \\
\text { time (ms) }\end{array}$ \\
\hline $\mathrm{HC}$ & $1.4(0.5)$ & 3431 (1654) & $1.2(0.4)$ & $2557(2032)$ \\
\hline VMF damage & $1.7(0.9)$ & 2504 (664) & $1.0(0.1)$ & 1895 (615) \\
\hline FC & $1.7(0.9)$ & 3123 (1255) & $1.1(0.3)$ & 1647 (702) \\
\hline
\end{tabular}

There was no significant group effect on reaction times in the five-fribble trials $\left(F_{(2,45)}=0.61, p=0.55, \eta_{\mathrm{p}}^{2}=0.03\right)$.

Attribute-value associations were retained very well over a 10 min delay, and there was no effect of group on accuracy (one-way ANOVA, $F_{(2,45)}=0.49, p=0.61, \eta_{\mathrm{p}}^{2}=0.02$; Table 3$)$.

\section{Attribute-value learning}

All subjects learned the stimulus-value associations to criterion (>92\% accuracy) within four learning blocks in both conditions. Across groups, more learning blocks were needed to reach criterion in the configural compared with the elemental condition (mixed-measure ANOVA, $F_{(1,45)}=17.04, p<0.001, \eta_{\mathrm{p}}^{2}=0.28$ ), but there was no significant group-by-condition interaction $\left(F_{(2,45)}=1.67, p=0.199, \eta_{\mathrm{p}}^{2}=0.07\right.$; Table 4). One HC participant was an outlier with respect to reaction times during learning probes (mean: configural condition, $8947 \mathrm{~ms}$; elemental condition, $10,765 \mathrm{~ms}$ ). After removing this participant from the analysis, we found no main effect of group on reaction times (configural, $F_{(2,44)}=1.82, p=0.18, \eta_{\mathrm{p}}^{2}=0.08$; elemental, $F_{(2,44)}$ $\left.=1.29, p=0.28, \eta_{\mathrm{p}}^{2}=0.05\right)$. The participant with very slow responses nonetheless learned all fribble-value associations to criterion and was included in further analysis.

\section{Multiattribute value-based choices}

Having established that patients with frontal damage could discriminate fribbles, learn elemental and configural value associations, and retain this information across a 10 min delay as well as HC participants, we next assessed multiattribute value-based binary decisions in elemental and configural conditions. The elemental condition involved choices between all possible pairs of fribbles. In principle, half of these trials could be solved by considering the values of single attributes, rather than integrating the values of two attributes, as both options have a value-predicting attribute in common. Trials in which the options differed on both value-predictive attributes (two-attribute trials) require somehow combining the values of two attributes, and were analyzed separately from the single-attribute trials. For the purposes of analysis, we summed the trained attribute values, but the identical relative value orderings would emerge from averaging these values, or from trading off the values of each individual attribute.

The values learned during training systematically influenced choice in both conditions (Fig. 3). GEEs were used to quantify the 
A

B

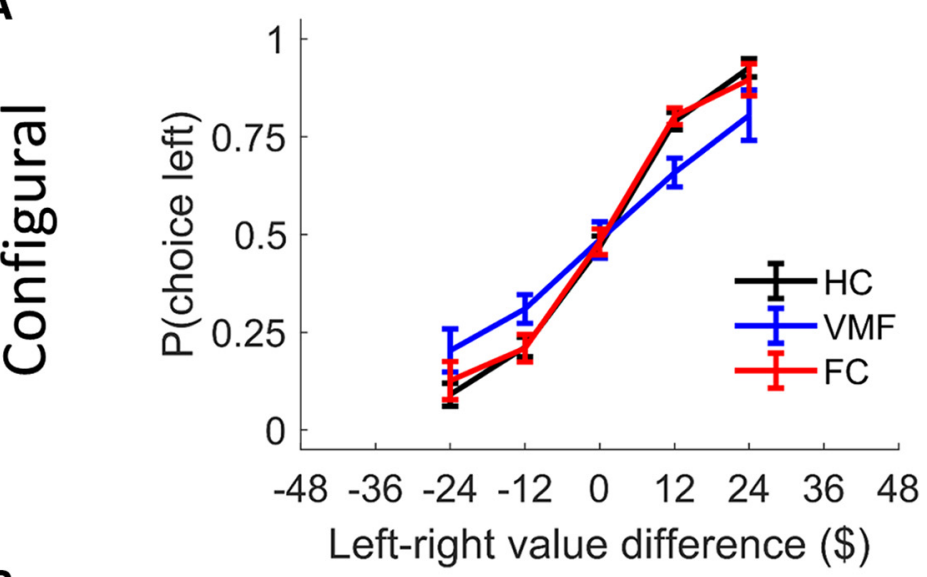

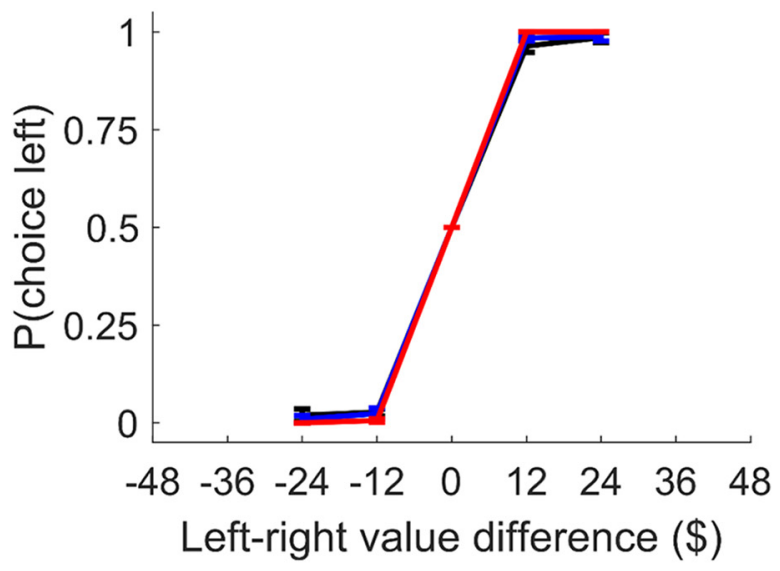

C
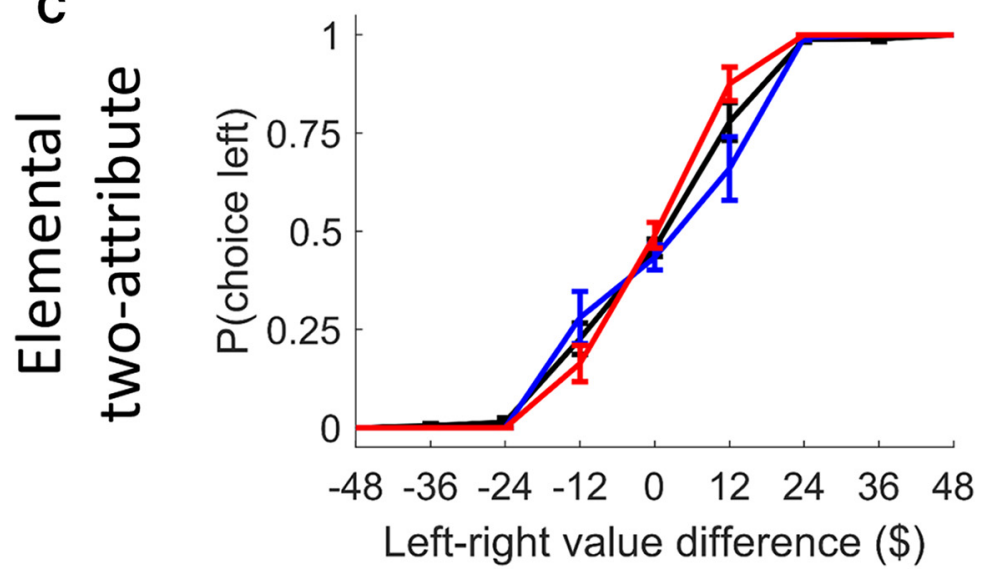

Figure 3. A-C, Probability of choosing the left option as a function of the relative value of the left and right options in the configural $(\boldsymbol{A})$ and elemental condition divided in single-attribute $(\boldsymbol{B})$ and two-attribute trials $(\boldsymbol{C})$. Error bars indicate SEM.

weakly predicted by option value difference (interaction OR, 0.58 ; 95\% CI, 0.39$0.84 ; p=0.004)$. In contrast, the choices of the FC group were influenced by option value difference to a similar degree as the HC group (no significant interaction between group and value; OR, 0.94; 95\% CI, $0.61-1.43 ; p=0.77)$.

In the elemental condition, the VMF group's choices were influenced by option value difference to a similar extent as the $\mathrm{HC}$ group in both the single-attribute (interaction OR, $1.57 ; 95 \% \mathrm{CI}, 0.34-7.06$; $p=0.56$ ) and two-attribute trials (interaction OR, 0.93; 95\% CI, 0.64-1.37; $p=$ $0.73)$. Value difference was a significantly stronger predictor of choice in the FC group compared with the $\mathrm{HC}$ group in the single-attribute (interaction OR, 17.20; 95\% CI, 3.52-84.07; $p<0.001)$ and the two-attribute trials (interaction OR, 1.64; 95\% CI, 1.03-2.59; $p=0.04$ ).

As can be seen in Figure 3, the range of value differences was greater in the twoattribute trials of the elemental condition compared with that of the single-attribute trials, and of the configural condition. Because greater value differences are generally associated with easier decisions, and all groups performed at ceiling at the extreme value differences (Fig. $3 C$ ), we restricted the analysis to the two-attribute trials within the same value-difference range as the other conditions. We again found no significant difference between the $\mathrm{HC}$ and the VMF groups (interaction OR, 0.87; 95\% CI, 0.57-1.34; $p=0.53$ ), and value difference tended to be a marginally better predictor of choice in the FC group compared with the HC group (interaction OR, $1.61 ; 95 \% \mathrm{CI}, 0.99-2.64$; $p=0.06)$.

We next asked whether group differences in the influence of value on choice were reflected in significant differences in choice accuracy, defined as the percentage of trials in which the highest value option was chosen. As depicted in Figure $4 A$, there was a significant main effect of group on accuracy in the configural condition (one-way ANOVA, $F_{(2,45)}=4.95$, $\left.p=0.01, \eta_{\mathrm{p}}^{2}=0.18\right)$. Post hoc tests with

extent to which choices were predicted by the difference in option values, trial by trial, and to test whether this differed by frontal lesion group. Across groups, choices were significantly predicted by the option value difference in both the configural [odds ratio (OR), 2.98; 95\% CI, 2.50-3.56; $p<0.0001$ ] and elemental conditions (single-attribute OR, 31.35; 95\% CI, 14.5267.70; $p<0.0001$; two-attribute OR, 5.47; 95\% CI, 4.54-6.60; $p<0.0001)$.

The group-by-value interaction was then added to the model, with $\mathrm{HC}$ as the reference group. Compared with the HC group, the VMF group's choices in the configural condition were more
Bonferroni correction for multiple comparisons revealed that the VMF group was less accurate than both the $\mathrm{HC}(p=0.01)$ and FC groups $(p=0.04)$, whereas the FC and HC groups were not significantly different $(p=1.0)$. There was no significant effect of group on reaction time (one-way ANOVA, $F_{(2,45)}=1.37, p=0.26, \eta_{\mathrm{p}}^{2}=0.06$; Fig. $3 C$ ) in the configural condition. In contrast, there was no significant effect of group on accuracy (one-way ANOVA, $F_{(2,45)}=2.47, p=0.10, \eta_{\mathrm{p}}^{2}=$ $0.10)$ or reaction time $\left(F_{(2,45)}=0.29, p=0.75, \eta_{\mathrm{p}}^{2}=0.01\right)$ in the elemental condition. 
Separating the elemental condition by trial types, we found that, across groups, participants were less accurate in twoattribute trials compared with singleattribute trials (mixed-measures ANOVA, $F_{(1,45)}=57.1, p<0.0001, \eta_{\mathrm{p}}^{2}=0.56$; Fig. $4 B)$. There was a trending but nonsignificant effect of group $\left(F_{(2,45)}=3.18, p=\right.$ $\left.0.06, \eta_{\mathrm{p}}^{2}=0.12\right)$ on accuracy, and no significant interaction between trial type and group $\left(F_{(2,45)}=2.24, p=0.12\right.$, $\left.\eta_{\mathrm{p}}^{2}=0.09\right)$.

Reaction times were longer in the twoattribute trials $\left(F_{(1,45)}=46.03, p<\right.$ 0.0001, $\eta_{\mathrm{p}}^{2}=0.50$; Fig. $\left.4 D\right)$. Again, there was no significant effect of group $\left(F_{(2,45)}\right.$ $\left.=0.29, p=0.75, \eta_{\mathrm{p}}^{2}=0.01\right)$ and no interaction between group status and trial type $\left(F_{(2,45)}=1.37, p=0.26, \eta_{\mathrm{p}}^{2}=0.06\right)$. In summary, VMF damage had no effect on decision accuracy between objects based on elemental values, whether for objects distinguished by a single attribute value or by two attribute values.

While there was a group effect in the configural but not in the elemental condition, when the two conditions were included in the same statistical model, there was no significant group-by-condition interaction (mixed-measures ANOVA, $\left.F_{(2,45)}=2.37, p=0.11, \eta_{\mathrm{p}}^{2}=0.10\right)$. However, many trials in the elemental condition did not require two attributes to be considered. We therefore undertook an additional, exploratory analysis focusing on the trials that were most similar in attribute-processing requirements across the two conditions, i.e., the 25 elemental condition trials where both attributes had to be considered to correctly assess value, and the 48 configural trials matched with these elemental trials on value difference between options. The difference in accuracy in these trials, for each subject, was then calculated (Fig. $4 E$ ), and this relative performance index was compared across groups. We found that the configural-elemental accuracy difference did not differ significantly from zero in the VMF (Wilcoxon signed rank test, $Z=$ $-0.13 p=0.90)$, HC $(Z=0.21, p=0.84)$ or FC groups $(Z=$ $-1.47, p=0.14)$, indicating that accuracy was similar across conditions. There was no effect of group on accuracy difference (Kruskal-Wallis H test, $\chi_{(2)}^{2}=0.88, p=0.64$ ). As is evident in Figure $4 E$, the variance in this subset of trials is high, particularly in the $\mathrm{HC}$ and VMF groups, limiting power to detect differences with this exploratory analysis, if present.

\section{Discussion}

We provide evidence that VMF damage impairs value-based decisions between novel multiattribute objects when overall value is predicted by the configuration of two attributes. This finding was specific to VMF damage: damage to other frontal regions did not impair value-based choices when overall value was predicted by attribute configuration. We did not find evidence that VMF damage impairs decisions between options when individual attributes are
B
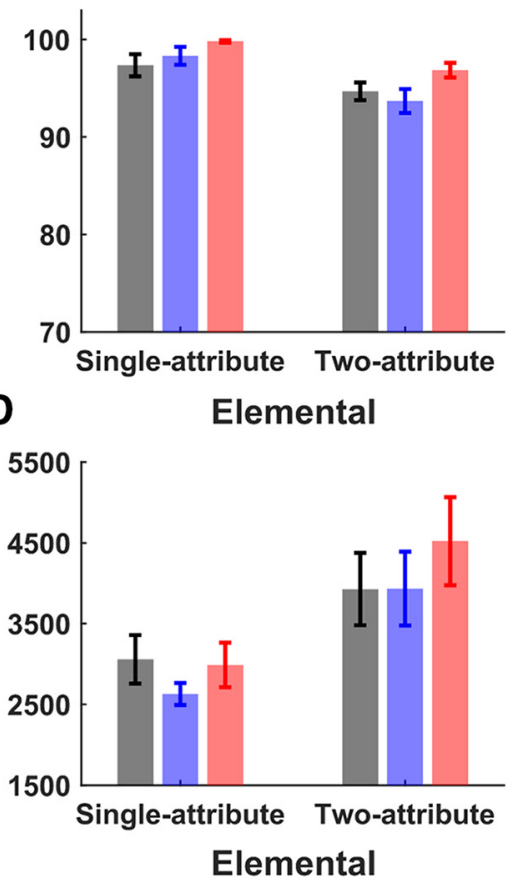

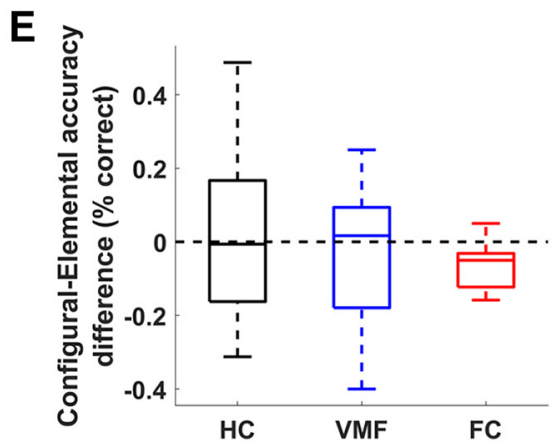

Figure 4. Multiattribute decision task performance. $\boldsymbol{A}, \boldsymbol{B}$, Choice accuracy for the $(\boldsymbol{A})$ configural and $(\boldsymbol{B})$ elemental condition. $\boldsymbol{C}$, $\boldsymbol{D}$, Reaction times for the $(\boldsymbol{C})$ configural and $(\boldsymbol{D})$ elemental condition. Error bars indicate $S E M,{ }^{*} p<0.05$. $\boldsymbol{E}$, Difference in accuracy between configural and elemental difficulty-matched trials. Distributions shown with median and quartiles.

independently predictive of value, either when value is assessed based on a single attribute, or when two attribute values are combined to make an optimal choice.

These findings argue that the VMF is involved in assessing the holistic value of multiattribute objects. This is the first direct evidence that the VMF plays a critical role in decisions based on value information provided by the conjunction of individual attributes, each of which is uninformative on its own. The results complement previous work from our laboratory showing that VMF damage leads to the neglect of some value-predictive information in complex real-world objects (faces, art; Xia et al., 2015; Vaidya et al., 2018). The present observations raise the possibility that such information may be neglected because it relies more heavily on configural processing. This might also explain the recent observation that VMF damage impairs value-based multiattribute decisions between social "objects" (spouses), but not between nonsocial objects (houses; Bowren et al., 2018), given the evidence that processing of social stimuli, such as faces, is fundamentally configural (Farah, 1996).

This framework may be more generally useful for guiding the growing neuroeconomics literature on multiattribute decision- 
making. The neural circuits involved may depend on whether options are presented in ways that encourage holistic or attributelevel processing. Furthermore, it is possible that attributes extrinsic to an object (such as quantity) may influence value through different mechanisms than intraoption attributes (such as taste or beauty; de Berker et al., 2019). We suggest that efforts to link research about object-processing and multiattribute decision processes (Bettman et al., 1998) may be helpful in advancing our understanding of how the brain makes value-based choices among real-world objects.

Although the present study was not designed to study valuebased learning, it is notable that the learning measures we collected suggest that the VMF is not required to learn configural whole-object values through feedback. Configural learning has been shown to rely on the hippocampus (Rudy and Sutherland, 1995), and configural reinforcement learning has been related to the functional coupling between the hippocampus and striatum (Duncan et al., 2018). Learning to choose objects based on configuration in nonhuman primates is spared after transection of the uncinate fasciculus, disrupting the direct connections between the prefrontal cortex and the temporal lobe (Gutnikov et al., 1997), which is consistent with our finding here that VMF damage does not substantially disrupt such learning in humans. As discussed above, the VMF becomes critical when configural object values must be compared with guide choice.

Decisions in the elemental condition could in principle be achieved by option-based or attribute-based strategies, either by integrating attribute values within options and then comparing the compound values, or by comparing individual attribute values. Extensive research has shown that further strategies may be engaged within these broad approaches to such decisions (e.g., trade-offs, elimination-by-attribute; Bettman et al., 1998). We cannot address which strategies might have been used here, but prior work on explicitly multiattribute (elemental) choices where attribute information is presented in tabular format has demonstrated that VMF damage affects these processes, biasing toward simpler, within-option valuation rather than cross-option comparison strategies (Fellows, 2006).

The present work clearly shows that VMF damage does not impair learning or choices based on single value-predictive attributes, when those attribute values are explicitly trained. Could the VMF also play a role in multiattribute decisions involving the integration of independent attribute values? Previous fMRI research has shown that activity in the VMF tracks the value of items composed of multiple independently value-predictive attributes (Kahnt et al., 2011; Lim et al., 2013). Our findings suggest that an intact VMF is not required for choice in such conditions. However, the analysis directly comparing performance on the subset of trials with the most similar attribute processing and value-difference requirements across conditions did not demonstrate a group-by-condition interaction, and had limited statistical power. Thus, we cannot exclude the possibility that the VMF is required when independent attribute values must be traded off to make an optimal choice, at least under some conditions. Further work is needed on this question.

Participants with VMF damage could readily discriminate between complex objects in a control task that relied on configural object representations, ruling out the possibility that the observed impairment in decisions based on configural value was due to perceptual deficits. Configural object perception has been shown to rely on the perirhinal cortex, an MTL region closely related to the hippocampus. Damage to the perirhinal cortex selectively impairs object discrimination when it relies on attri- bute configurations (Barense et al., 2007; Bartko et al., 2007). In addition, patterns of BOLD activity in this area relate to complex objects held in working memory, but not their separate parts, and are relatively insensitive to viewpoint (Erez et al., 2016), arguing that the perirhinal cortex represents the identity of objects independent of changes in physical characteristics. Our findings suggest that the role of the VMF can be understood in similar terms: i.e., the VMF is crucial in developing predictive value representations when attributes on their own are ambiguous or separately uninformative. We speculate that perirhinal cortex interactions with the VMF may be important for predicting object values based on attribute configurations.

This proposal aligns with other recent efforts to understand how the prefrontal cortex and the MTL interact more generally. Synthesizing the effects of human hippocampus and VMF damage in a variety of cognitive domains, McCormick and colleagues argued that the VMF plays a supervisory role over the hippocampus in initiating and organizing episodic memory retrieval (McCormick et al., 2018). This interpretation mainly stems from studies addressing autobiographical memories and mental scene construction, with so far little causal evidence available with respect to memory for complex objects of the kind commonly featured in neuroeconomics research and everyday decisions. There is some evidence for hippocampus-VMF interactions during value-based decision. One fMRI study found that when imagining the consumption of novel foods composed of two familiar ingredients, both the VMF and hippocampus tracked the construction of the compound value (Barron et al., 2013). Interestingly, the results held after controlling for the value of each element, indicating that the compound value was distinct from the linear combination of the elements (i.e., configural). Given the role of the MTL in configural processing and our findings here, the putative supervisory role of the VMF over the MTL in episodic memory retrieval may extend to subjective value construction for multiattribute objects, particularly when individual attribute-value relationships are insufficiently informative.

An alternative account suggests that the VMF (OFC, specifically) encodes the latent (not directly observable) variables of a task to determine the current goals, i.e., representing a cognitive map of task states (Schuck et al., 2016). Task-state representations in the OFC, as they have been studied so far, are also compatible with a role of the VMF in configural decisions. The term "configural" implies that each observable element is not informative alone. Value is instead inferred from the association between elements, with each element being part of multiple associations. Similarly, in Schuck and colleagues (2016), task states were defined by the configuration of task variables, with each unique variable being part of many states. Further work is needed to establish whether these two accounts of the role of the VMFone emerging from computational views of goal states, the other from complex object processing - reflect the same underlying processes.

This study has limitations. While all patients included in this study had well characterized focal lesions, disruption of underlying white matter tracts (fibers of passage) can affect regions distant from the lesion site (Rudebeck et al., 2013). Converging evidence, especially from nonhuman primates where more selective lesions are possible, would be helpful in establishing whether effects are caused by white matter disruption, cortical damage, or both. We have too few patients with left-hemisphere VMF damage to establish whether the observed effects are lateralized to the right hemisphere. The task also had limited power to assess elemental multiattribute choices requiring trade-offs, limiting con- 
clusions about whether the VMF is also involved under those conditions. Interestingly, we found preliminary evidence that patients with damage affecting other frontal regions had difficulty with such trials, perhaps reflecting the role of the lateral and dorsomedial prefrontal cortices in attentional set-shifting (Dias et al., 1996; Vaidya and Fellows, 2016). Further work on the prefrontal mechanisms of individual attribute-value trade-offs in multiattribute choice is needed. Finally, task order was fixed, because we were most interested in configural processing and wanted to avoid introducing competition between elemental and configural strategies or task sets through the training procedures. For the same reason, we minimized attentional demands in the elemental training condition. All these design choices may be relevant to the pattern of observed effects.

In conclusion, these findings do not support the view that the VMF is generically necessary for tracking or comparing value information in a common currency. Under many real-world conditions, the value of complex objects might be better understood as a property that emerges from interactions between perception and memory processes, and that critically relies on the VMF when the value is ambiguous and embedded in the relational content among the parts that compose the whole.

\section{References}

Andrews-Hanna JR, Smallwood J, Spreng RN (2014) The default network and self-generated thought: component processes, dynamic control, and clinical relevance. Ann N Y Acad Sci 1316:29-52.

Barense MD, Gaffan D, Graham KS (2007) The human medial temporal lobe processes online representations of complex objects. Neuropsychologia 45:2963-2974.

Barron HC, Dolan RJ, Behrens TE (2013) Online evaluation of novel choices by simultaneous representation of multiple memories. Nat Neurosci 16:1492-1498.

Barry TJ, Griffith JW, De Rossi S, Hermans D (2014) Meet the Fribbles: novel stimuli for use within behavioural research. Front Psychol 5:103.

Bartko SJ, Winters BD, Cowell RA, Saksida LM, Bussey TJ (2007) Perirhinal cortex resolves feature ambiguity in configural object recognition and perceptual oddity tasks. Learn Mem 14:821-832.

Bartra O, McGuire JT, Kable JW (2013) The valuation system: a coordinatebased meta-analysis of BOLD fMRI experiments examining neural correlates of subjective value. Neuroimage 76:412-427.

Basten U, Biele G, Heekeren HR, Fiebach CJ (2010) How the brain integrates costs and benefits during decision making. Proc Natl Acad Sci U S A 107:21767-21772.

Benton A, Hamster K, Sivan A (1989) Multilingual Aphasia Examination. Iowa City, IA: AJA Associates.

Bettman JR, Luce MF, Payne JW (1998) Constructive consumer choice processes. J Consum Res 25:187-217.

Bower GH, Karlin MB (1974) Depth of processing pictures of faces and recognition memory. J Exp Psychol 103:751-757.

Bowren MD, Croft KE, Reber J, Tranel D (2018) Choosing spouses and houses: impaired congruence between preference and choice following damage to the ventromedial prefrontal cortex. Neuropsychology 32:280303.

Brainard DH (1997) The psychophysics toolbox. Spat Vis 10:433-436.

Bussey TJ, Saksida LM, Murray EA (2005) The perceptual-mnemonic/feature conjunction model of perirhinal cortex function. Q J Exp Psychol B $58: 269-282$.

de Berker AO, Kurth-Nelson Z, Rutledge RB, Bestmann S, Dolan RJ (2019) Computing value from quality and quantity in human decision-making. J Neurosci 39:163-176.

De Renzi E, Vignolo LA (1962) The token test: a sensitive test to detect receptive disturbances in aphasics. Brain 85:665-678.

Dias R, Robbins TW, Roberts AC (1996) Dissociation in prefrontal cortex of affective and attentional shifts. Nature 380:69-72.

Duncan K, Doll BB, Daw ND, Shohamy D (2018) More than the sum of its parts: a role for the hippocampus in configural reinforcement learning. Neuron 98:645-657.e6.

Eichenbaum H (2017) Prefrontal-hippocampal interactions in episodic memory. Nat Rev Neurosci 18:547-558.

Erez J, Cusack R, Kendall W, Barense MD (2016) Conjunctive coding of complex object features. Cereb Cortex 26:2271-2282.

Farah MJ (1996) Is face recognition 'special'? Evidence from neuropsychology. Behav Brain Res 76:181-189.

Fellows LK (2006) Deciding how to decide: ventromedial frontal lobe damage affects information acquisition in multi-attribute decision making. Brain 129:944-952.

Gluth S, Sommer T, Rieskamp J, Büchel C (2015) Effective connectivity between hippocampus and ventromedial prefrontal cortex controls preferential choices from memory. Neuron 86:1078-1090.

Grober E, Sliwinski M (1991) Development and validation of a model for estimating premorbid verbal intelligence in the elderly. J Clin Exp Neuropsychol 13:933-949.

Gutnikov SA, Ma YY, Gaffan D (1997) Temporo-frontal disconnection impairs visual-visual paired association learning but not configural learning in macaca monkeys. Eur J Neurosci 9:1524-1529.

Kahnt T, Heinzle J, Park SQ, Haynes JD (2011) Decoding different roles for vmPFC and dlPFC in multi-attribute decision making. Neuroimage 56:709-715.

Levy DJ, Glimcher PW (2012) The root of all value: a neural common currency for choice. Curr Opin Neurobiol 22:1027-1038.

Lezak MD, Howieson DB, Loring DW, Fischer JS (2012) Neuropsychological Assessment. New York: Oxford UP.

Lim SL, O'Doherty JP, Rangel A (2013) Stimulus value signals in ventromedial PFC reflect the integration of attribute value signals computed in fusiform gyrus and posterior superior temporal gyrus. J Neurosci 33: $8729-8741$

McCormick C, Ciaramelli E, De Luca F, Maguire EA (2018) Comparing and contrasting the cognitive effects of hippocampal and ventromedial prefrontal cortex damage: a review of human lesion studies. Neuroscience 374:295-318.

Olson IR, Page K, Moore KS, Chatterjee A, Verfaellie M (2006) Working memory for conjunctions relies on the medial temporal lobe. J Neurosci 26:4596-4601.

Padoa-Schioppa C, Assad JA (2006) Neurons in the orbitofrontal cortex encode economic value. Nature 441:223-226.

Rorden C, Brett M (2000) Stereotaxic display of brain lesions. Behav Neurol 12:191-200.

Rudebeck PH, Saunders RC, Prescott AT, Chau LS, Murray EA (2013) Prefrontal mechanisms of behavioral flexibility, emotion regulation and value updating. Nat Neurosci 16:1140-1145.

Rudy JW, Sutherland RJ (1995) Configural association theory and the hippocampal formation: an appraisal and reconfiguration. Hippocampus 5:375-389.

Schuck NW, Cai MB, Wilson RC, Niv Y (2016) Human orbitofrontal cortex represents a cognitive map of state space. Neuron 91:1402-1412.

Suzuki S, Cross L, O'Doherty JP (2017) Elucidating the underlying components of food valuation in the human orbitofrontal cortex. Nat Neurosci 20:1780-1786.

Vaidya AR, Fellows LK (2016) Necessary contributions of human frontal lobe subregions to reward learning in a dynamic, multidimensional environment. J Neurosci 36:9843-9858.

Vaidya AR, Sefranek M, Fellows LK (2018) Ventromedial frontal lobe damage alters how specific attributes are weighed in subjective valuation. Cereb Cortex 28:3857-3867.

Von Der Heide RJ, Skipper LM, Klobusicky E, Olson IR (2013) Dissecting the uncinate fasciculus: disorders, controversies and a hypothesis. Brain 136:1692-1707.

Williams P, Simons DJ (2000) Detecting changes in novel, complex threedimensional objects. Vis Cogn 7:297-322.

Xia C, Stolle D, Gidengil E, Fellows LK (2015) Lateral orbitofrontal cortex links social impressions to political choices. J Neurosci 35:8507-8514.

Zigmond AS, Snaith RP (1983) The hospital anxiety and depression scale. Acta Psychiatrica Scandinavica 67:361-370. 